\title{
Fetal growth restriction - pathophysiology, diagnosis and management of uteroplacental insufficiency before 34 weeks
}

\author{
Tiran Dias ${ }^{1}$ \\ Sri Lanka Journal of Obstetrics and Gynaecology 2012; 34: 128-130
}

\section{Pathophysiology}

Placental trophoblastic cell invasion into the media of the maternal uterine spiral arteries is responsible for decreasing blood flow resistance in the maternal compartment of the placenta. An abnormal invasion of placental trophoblast will result in progressive resistance in the utero-placental system and reduction in blood flow to the fetus. Successful trophoblast invasion results in loss of early diastolic notching in the uterine artery Doppler waveform by the end of first trimester in most women followed by successive decline in the Doppler index throughout gestation.

Under normal circumstances nearly $30 \%$ of blood in umbilical vein is shunted towards the foramen ovale through the ductus venosus (DV) and remaining $70-80 \%$ continues to the liver. Reduced UV volume flow in uteroplacental insufficiency leads to increased DV-shunting away from the liver towards the heart. Liver glycogen storage and glucose-insulin-IgF growth axis is diminished subsequent to this venous redistribution. This leads to progressive decrease in liver size. Reduced fetal abdominal circumference is the first clinical sign before the estimated fetal weight falls below the 10th percentile.

Umbilical artery Doppler starts to become abnormal when placental villous obliteration affects approximately one-third of the villous vasculature. Thereafter, significant relative decrease in oxygen and increase in fetal blood pressure produce a consistent decrease of the middle cerebral artery Doppler index. Umbilical artery diastolic flow becomes absent or reverse when villous obliteration is more than 50\% and risk of fetal hypoxia and acidosis is progressively increasing with further obliteration of villous structure. A decline in cardiac performance contributes to an elevation of central venous pressure and decreasing forward cardiac function. Under these

\footnotetext{
${ }^{1}$ Consultant Obstetrician and Gynaecologist, General Hospital, Ampara.
}

circumstances ductus venosus 'a' wave becomes absent or reverse and pulsation of umbilical vein appears.

\section{Diagnosis}

Fetal growth restriction (FGR) is often equated to small for gestational age (SGA). SGA refers to fetal weight below the 10th centile for particular gestational age and it constitutes both FGR and constitutionally small fetuses. The best antenatal test for fetal growth restriction is serial assessment of fetal abdominal circumference $(\mathrm{AC})$ and diagnosis can be confirmed when the $A C$ is crossing the centiles. Single AC measurement below the 5 th centile with abnormal Doppler studies and reduced amniotic fluid index can also be used to diagnose FGR (Figure 1 and 2). Use of customized growth charts which consider maternal characteristics and parity helps in differentiating SGA and FGR. Ultrasound biometry should not be repeated more often than 2 weeks as early interval would bias with inter and intra observer variation.

\section{Management}

Gestational age and birthweight are the primary determinants of the outcome as iatrogenic early delivery carries a higher rate of neonatal complications, whereas delayed delivery carries the risk of unintended stillbirth. Gestational age at onset has important impact on the clinical decision making.

\section{Early onset FGR before 34 weeks}

In early onset FGR before 34 weeks gestation, neonates show higher incidence of morbidities than appropriately grown counterpart. It has been realized that the disease progression in early onset FGR can be monitored more objectively. The typical pattern of deterioration progresses from escalating abnormalities in umbilical artery (UA) and venous Doppler parameters to abnormal biophysical parameters. The purpose of fetal wellbeing assessment is to recognize early fetal compromise and timely delivery. Therefore, monitoring should be adjusted according to the disease acceleration and interventional threshold. 

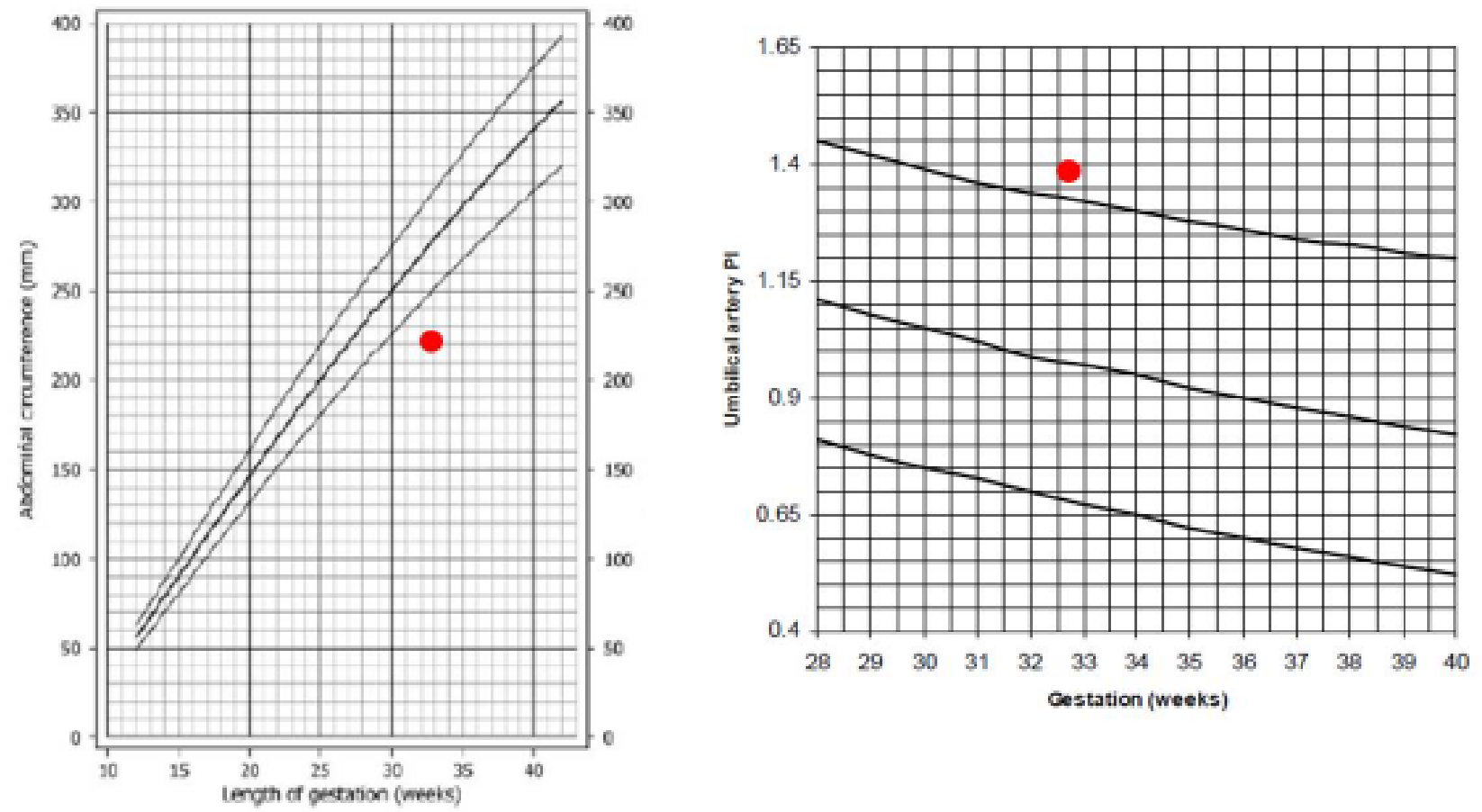

Figure 1. Fetal abdominal circumference is the most important fetal biometry in diagnosing FGR. Single measurement below the $5^{\text {th }}$ centile with abnormal Doppler can be used to diagnose FGR in well dated pregnancies.
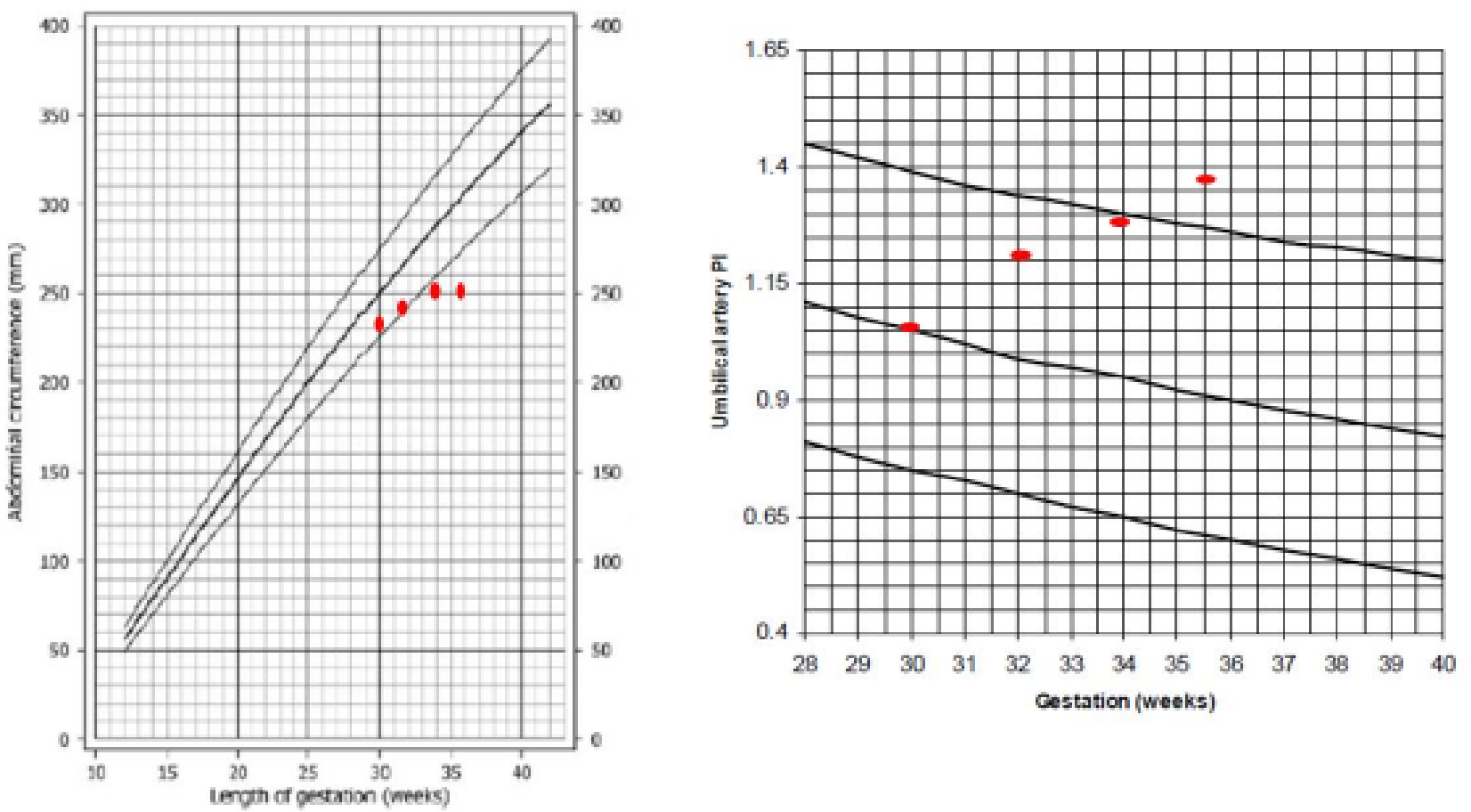

Figure 2. Diagnosis of FGR can be made on the basis of crossing centiles. Growth velocity is reduced over the time with abnormal UA Doppler trend. 
It is recognized that delivery should be considered in case of absent end diastolic flow in the UA after 34 weeks. Weekly follow-up is appropriate in the presence of positive diastolic flow in the UA before 34 weeks. More frequent fetal wellbeing assessment is needed in the loss or reversal of end diastolic flow in UA, increasing DV Doppler indices, and decreasing amniotic fluid volume as these findings would indicate disease acceleration. Fetal hypoxemia and acidaemia is likely when DV pulsatility index (PI) is abnormal and delivery is indicated in such case after 32 weeks. There is no established guideline in managing FGR before 32 weeks. Short term variability in computerize cardio-tocograph or presence/absence of 'a' wave in DV can be used to guild the timing of delivery before 32 weeks. This approach is currently being evaluated in the trial of umbilical and fetal flow in Europe.
Late onset FGR after 34 weeks

Intervention threshold is less challenging in late onset FGR because gestational age plays a minor role in determining outcome. However, the emphasis should be on detection and determination of surveillance intervals. In late-onset FGR, the typical progression of arterial to venous Doppler abnormalities is not seen. As placental vascular dysfunction is less severe, a decreased cerebroplacental Doppler ratio, with either normal or only minimally elevated UA Doppler indices, may be observed. This is followed by preferential blood flow to the brain and a decreased MCA Doppler index that may occur as an isolated finding without a preceding increase in the UA Doppler index. MCA Doppler index and rate of progression on UA Doppler index can be used to guide the timing of delivery in late onset FGR (Figure 3).
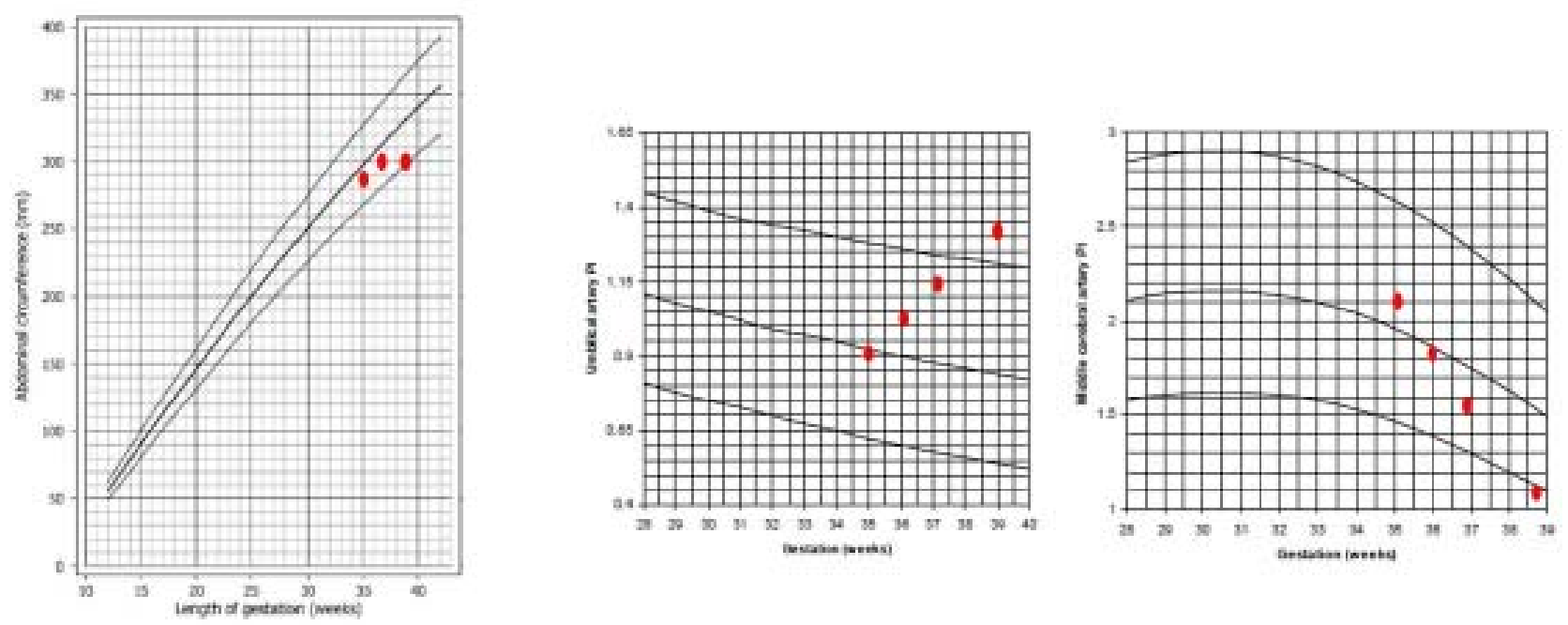

Figure 3. Decision of delivery should be based on MCA Doppler and UA Doppler trend in FGR after 34 weeks.

\section{References}

1. Thompson JL, Kuller JA, Rhee EH. Antenatal surveillance of fetal growth restriction. Obstet Gynecol Surv 2012; 67(9): 554-65.

2. Baschat AA. Fetal growth restriction - From observation to intervention. J Perinat Med 2010; 38: 239-46.

3. Lees C, Baumgartner H. The TRUFFLE study - a collaborative publicly funded project from concept to reality: how to negotiate an ethical, administrative and funding obstacle course in the European Union. Ultrasound Obstet Gynecol 2005; 25: 105-7.

4. Hecher K, Bilardo CM, Stigter RH, Ville Y, Hackel “ oer BJ, Kok HJ, Senat MV, Visser GH. Monitoring of fetuses with intrauterine growth restriction: a longitudinal study. Ultrasound Obstet Gynecol 2001; 18: 564-70. 